\title{
Color Filter Arrays: Design and Performance Analysis
}

\author{
Rastislav Lukac, Member, IEEE, and Konstantinos N. Plataniotis, Senior Member, IEEE
}

\begin{abstract}
This paper describes the design of color filter arrays (CFAs) used in the consumer-grade digital camera, and analyses their influence on the performance of the demosaicking process. Of particular interest are RGB CFAs widely used in a single-sensor imaging pipeline. Different design characteristics of various image-enabled consumer electronic devices by the different manufacturers lead to the several arrangements of the color filters in the CFA, affecting both performance and computational efficiency of the demosaicking solution. Extensive experimentation, using ten $R G B$ CFAs and a universal demosaicking framework, reported in this paper indicates that the CFA has a great impact on both the objective and subjective (visual) quality of the demosaicked, full-color image. ${ }^{l}$
\end{abstract}

Index Terms - Image-enabled consumer electronics, singlesensor imaging, color filter array, demosaicking.

\section{INTRODUCTION}

COLOR filter array (CFA) is one of the most distinctive hardware elements in a single-sensor imaging pipeline [1]. The CFA is placed on top of the monochrome image sensor, usually a charge-coupled device (CCD) [2] or complementary metal oxide semiconductor (CMOS) [3] sensor, to acquire the low-resolution color information of the image scene. Each sensor cell has its own spectrally selective filter and thus, the acquired CFA data constitutes a mosaic-like monochrome image (Fig. 1a) [4]. Since the information about the arrangement of the color filters in the CFA is known from the camera manufacturers or it can be obtained using the Tagged Image File Format for Electronic Photography (TIFF-EP) [5], the gray-scale CFA image can be re-arranged as alowresolution color image (Fig. 1b) [4]. This is the initial operation in the demosaicking process [6]-[8] which uses the concept of spectral interpolation to estimate the missing color components and to produce a full-color image (Fig. 1c) [9].

The arrangement of the color filters in the CFA varies depending on the manufacturer [10]-[14]. Consumer electronic devices, such as various digital still and video cameras, imageenabled mobile phones, and wireless personal digital assistants (PDAs) thus naturally differ in the employed demosaicking solution. Different cost and implementation constraints are expected for a camera which stores the image in the CFA format and uses a companion personal computer to demosaick

The authors are with The Edward S. Rogers Sr. Department of ECE, University of Toronto, Toronto, Canada.

Corresponding Author: Dr. Rastislav Lukac, Multimedia Laboratory, Room BA 4157, The Edward S. Rogers Sr. Department of ECE, University of Toronto, 10 King's College Road, Toronto, Ontario, M5S 3G4, Canada (e-mail: lukacr@ieee.org, web: http://www.dsp.utoronto.ca/ lukacr)

Contributed Paper

Manuscript received August 30, 2005 (a)
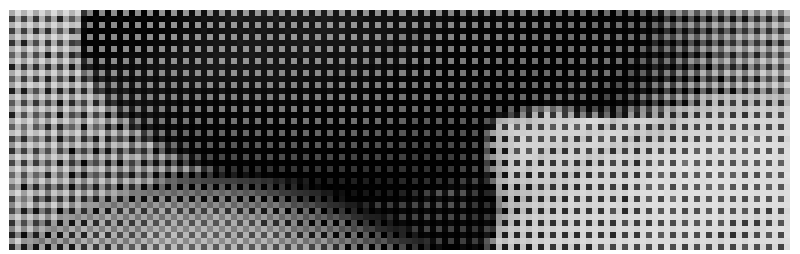

(b)

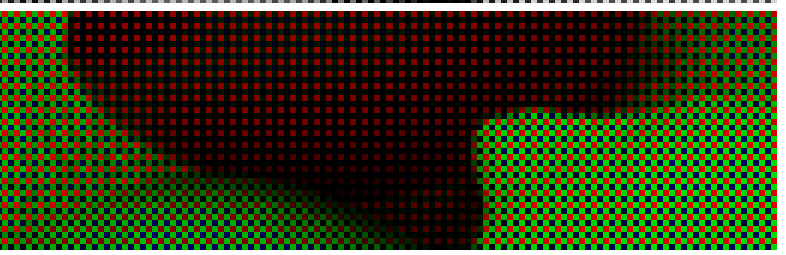

(c)

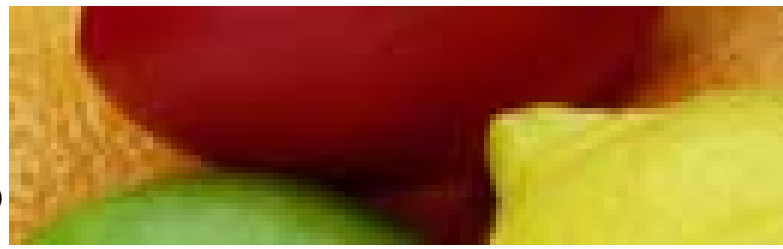

Fig. 1. Single-sensor imaging: (a) mosaic-like gray-scale CFA image, (b) color variant of the CFA image, (c) demosaicked full-color image.

the acquired image data, than for a camera which directly produces the demosaicked image. Other construction differences may result from the intended application (e.g. consumer photography, surveillance, astronomy).

Although mistakenly neglected in the research papers, the choice of the CFA critically influences the accuracy of the single-sensor imaging pipeline [12],[14]. Both the sharpness and the color appearance of the edges and fine details in the demosaicked image depend on the CFA layout in the edge area and its closest neighborhood. If signal structures in the captured image have size smaller than the sampling frequency of an arbitrary color band in the CFA, the demosaicking process usually results in various visual impairments such as aliasing, moire noise and color shifts [4],[8],[15]. Thus, the use of another CFA may eliminate the presence of artifacts in certain areas of the demosaicked image while degrading the image quality in other areas.

In this paper, the demosaicking performance is analyzed with respect to ten different types of the RGB CFA employed in the single-sensor imaging pipeline. In addition to the nine known CFAs with a periodic, pseudo-random or human visual system (HVS)-based structure, this paper introduces a new CFA which completes the available designs. To truly analyze the CFA efficiency, a universal demosaicking framework [14] is employed. Please note that although extensive research has been devoted to demosaicking of the images captured using the Bayer CFA (Fig. 2a) [10], there is no known work addressing the performance issues for other CFAs (Figs. 2b-j) in such a comprehensive and systematic way. 


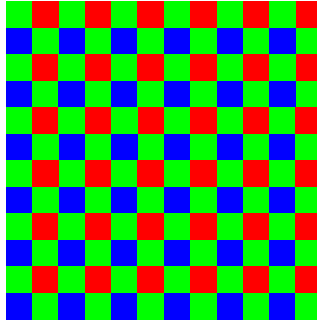

(a)

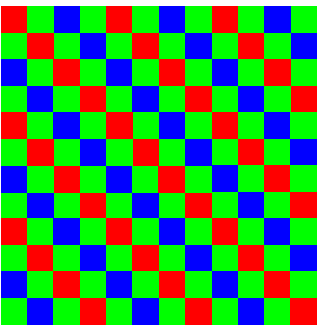

(f)

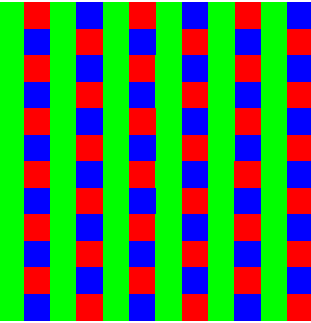

(b)

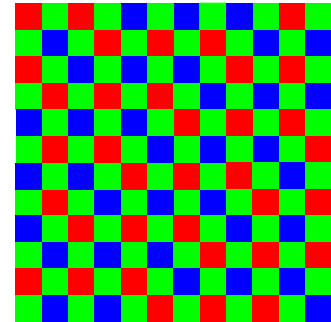

(g)

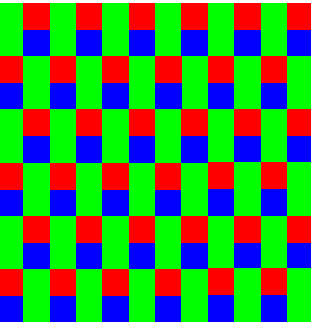

(c)

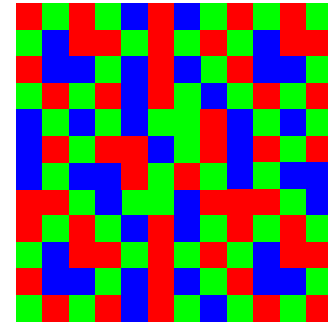

(h)

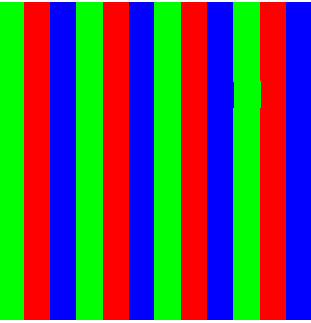

(d)

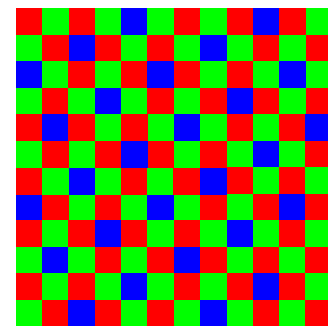

(i)

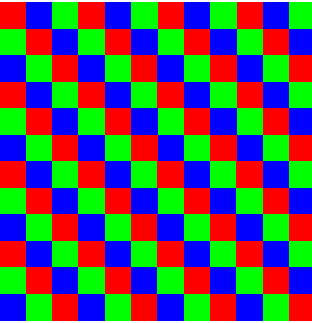

(e)

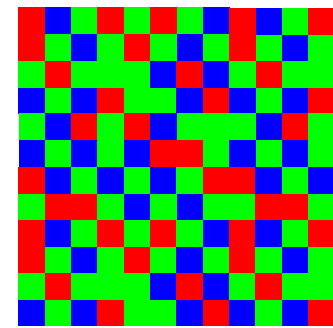

(j)

Fig. 2. RGB CFAs: (a) Bayer CFA [10], (b) Yamanaka CFA [11], (c) proposed here CFA, (d) vertical stripe CFA [12], (e) diagonal stripe CFA [12], (f) modified Bayer CFA [12], (g-i) pseudo-random CFA [12], (j) HVS-based CFA [13].

The rest of this paper is organized as follows. The RGB CFAs are introduced in Section II. Motivation and design characteristics are discussed in detail, and the brief description of the universal demosaicking framework suitable to process the CFA image captured using an arbitrary RGB CFA is included, as well. In Section III, the presented CFAs are tested using the universal demosaicking framework and various artificial and natural images. Evaluations of performance, both objective and subjective, are provided. Finally, conclusions are drawn in Section IV.

\section{COLOR FILTER ARRAY}

Both the design and performance characteristics of the CFA are essentially determined by the type of a color system and the arrangements of the color filters in the CFA [12],[14]. These two basic CFA features specify the construction requirements of the demosaicking solution, thus influencing its efficiency and cost.

\section{A. CFA Design Guidelines}

Today's color systems [4],[12] used in various CFA designs utilize: i) tri-stimulus color basis (RGB, YMC), ii) mixed primary/complementary colors (MGCY), and iii) four and more color concepts (e.g. RGB combined with white and/or color with shifted spectral sensitivity). Since the individual color filters are usually layers of transmitive (organic or pigment) dyes [16], the choice of dyes depends on the factors, such as ease of application, durability, and resistance to aggressive atmospheric conditions. Assuming light sensitivity as another criterion in the CFA design, the complementary or spectrally shifted color filters obtained by layering the dyes corresponding to the primary RGB colors (Fig. 3), are naturally less sensitive to the incoming light than the primary color filters obtained using a single-dye layer.
Focusing on the colorimetric properties, more accurate hue gamut is usually obtained by the CFAs based on mixed colors or various four-(or more) color concepts [12]. On the other hand, these designs may extremely increase the complexity of the demosaicking process [14]. In addition, the utilization of the mixed primary/complementary colors in the CFA often limits the useful range of the darker colors [12]. Since the images are commonly stored in the RGB color format and the tri-stimulus RGB system offers the way to acquire the image data in the required format, RGB CFAs constitute the most practical solution which may achieve the essential trade-off between the accuracy of the visual scene representation and the demosaicking complexity. For the same reason, the RGB CFAs are used throughout this paper.

Visual inspection of the RGB CFAs shown in Fig. 2 reveals that the arrangement of color filters in the array usually varies significantly. The difference in the CFA layout should be attributed to the effort of the camera manufacturers to obtain [12]: i) cost-effective image reconstruction, ii) immunity to color artifacts and color moiré, iii) robustness of the array to image sensor imperfections, and iv) immunity to optical/electrical cross talk between neighboring pixels.

The real-time processing constraints imposed on the digital camera usually require to simplify the demosaicking process as much as possible. This request is satisfied by the periodic CFAs (Figs. 2a-f), whereas the various pseudo-random CFAs (Figs. 2g-j) make the restoration process more complex due to their aperiodic nature. On the other hand, images captured using pseudo-random (or random) CFAs are usually more immune to color moiré effects [12]. Sensitivity of the array to color artifacts in the demosaicked image can be also reduced through the availability of the pixels' neighborhoods constituted by all three primary colors (Figs. 2d-f) and/or by allocating the larger amount of CFA locations to the G plane 


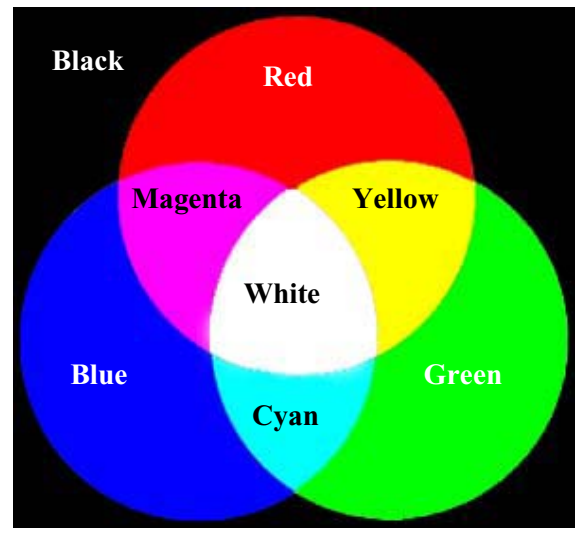

Fig. 3. Additive color mixing concept. Any spectrally shifted color can be obtained using the different amount of the three RGB primary colors.

(Figs. 2a-c,g,i,j). Since the frequency of the G color band is close to the peak of the human luminance frequency response [17],[18], privileging the $\mathrm{G}$ color filters in the CFA layout improves the perceived sharpness of the captured image.

Image sensor imperfections are usually observed along rows or columns of the sensor cells and thus, the CFAs shown in Figs. 2e,f should avoid the visual impairments resulting from the sensor defects. Immunity to optical/electrical cross talk between neighboring pixels can be increased by creating the CFA with the fixed number of neighbors corresponding to each of the three primary colors. This request is even more important due to the fact that diagonally located neighbors have a lower cross-talk contribution than the vertically or horizontally located neighbors [12], making the CFAs shown in Figs. 2g-j the worst solutions in terms of this criterion.

Since no CFA satisfies all design conditions, manufacturers usually select the CFA layout according to the type and resolution of the image sensor, camera optical system, image processing capabilities of the device, and the intended application. However, once the CFA layout is selected to acquire the CFA image data, the visual quality of the demosaicked full-color image depends on the ability of the demosaicking solution to overcome various spatial, structural and spectral constraints imposed on the single-sensor device during the image formation and color reconstruction.

\section{B. Universal Demosaicking Framework}

The acquired CFA image is a $K_{1} \times K_{2}$ gray-scale mosaic-like image $z: Z^{2} \rightarrow Z$ with the single scalar value $z_{(r, s)}$ located at each spatial location $(r, s)$. Operating along the row and column coordinates $r=1,2, \ldots, K_{1}$ and $s=1,2, \ldots, K_{2}$, respectively, the information about the $\mathrm{R}(k=1), \mathrm{G}(k=2)$, or $\mathrm{B}(k=3)$ color filters in the CFA can be stored using the location flags $d_{(r, s) k}$ obtained either from the CFA layout or using the TIFF-EP storage format [14]. Following the dimensions of the CFA image $z$, a $K_{1} \times K_{2}$ vectorial field $d: Z^{2} \rightarrow Z^{3}$ of the corresponding location flags $d_{(r, s) k}$ is initialized using the default value $d_{(r, s) k}=1$ to indicate the presence of the $k$-th color filter at the sensor location $(r, s)$, and using the flags set to $d_{(r, s) k}=0$ in all other cases. (a)
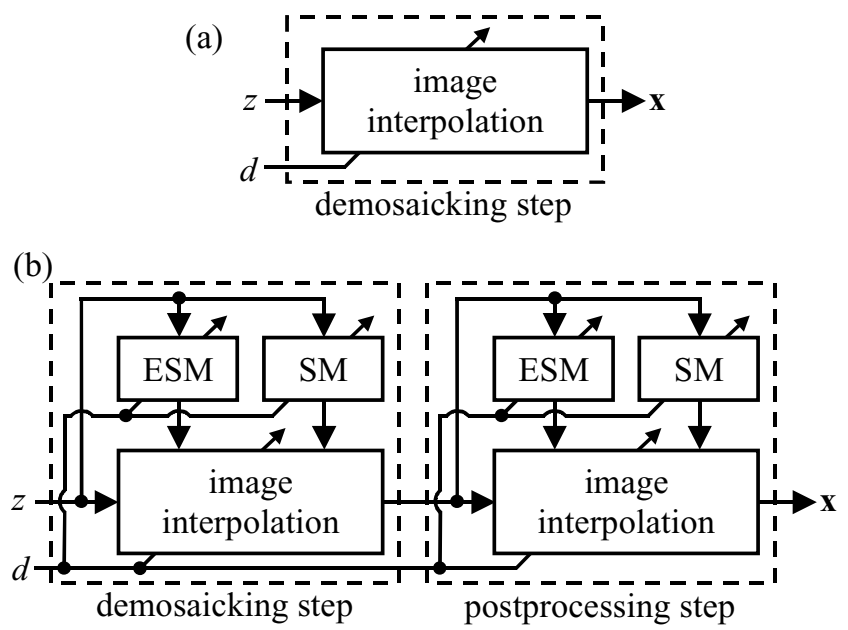

Fig. 4. Two basic solutions designed within the universal demosaicking framework [14]: (a) non-adaptive component-wise solution, (b) edge-sensing spectral model based solution with the postprocessor.

The CFA image $z$ is used as the input to a demosaicking solution which performs spectral interpolation [9] to obtain a $K_{1} \times K_{2}$ demosaicked full-color image $\mathbf{x}: Z^{2} \rightarrow Z^{3}$. The demosaicking process starts by re-arranging the CFA image (Fig. 1a) to its color variant (Fig. 1b). Using the location flags $d_{(r, s) k}$, the scalar CFA image data $z_{(r, s)}$ are transformed to the corresponding vectorial representation $\mathbf{x}_{(r, s)}=\left[x_{(r, s) k}, x_{(r, s) k}, x_{(r, s) k}\right]$ with $x_{(r, s) k}$ denoting the intensity in the $\mathrm{R}(k=1), \mathrm{G}(k=2)$, or $\mathrm{B}$ $(k=3)$ channel of the color image $\mathbf{x}$. Thus, the process produces the color vector $\mathbf{x}_{(r, s)}=\left[z_{(r, s)}, 0,0\right]$ for $d_{(r, s) 1}=1, \quad \mathbf{x}_{(r, s)}=\left[0, z_{(r, s)}, 0\right]$ for $d_{(r, s) 2}=1$, and $\mathbf{x}_{(r, s)}=\left[0,0, z_{(r, s)}\right]$ for $d_{(r, s) 3}=1$. Given the location flags $d_{(r, s) k}=0$ denoting the missing components in $\mathbf{x}_{(r, s)}$, the corresponding values of $x_{(r, s) k}$ are set equal to zero to denote their portion to the coloration of the color image $\mathbf{x}$ shown in Fig. $1 b$.

To produce a full-color image (Fig. 1c), the missing components are estimated at each spatial location $(r, s)$ from the available neighboring components using the concept of image interpolation [9]. Using a $3 \times 3$ sliding window instead of the specialized shape masks known from the Bayer CFA-based demosaicking solutions (e.g. [1],[4],[6]-[8],[15]-[22]), the universal demosaicking framework [14] is directly applicable to an arbitrary CFA shown in Fig. 2. By localizing the flags $d_{(r, s) k}=0$ used to indicate the spatial location $(r, s)$ in the $k$-th color channel to be demosaicked and utilizing the control mechanism to prevent from operating in areas which lack adequate input information, the framework obtains the essential flexibility and independence from the CFA layout. In addition, the framework offers a number of design and processing options to demosaick the acquired CFA data, ranging from the cost-effective non-adaptive component-wise solutions (Fig. 4a) to sophisticated solutions (Fig. 4b) which use the edge-sensing mechanism (ESM), the spectral model (SM) and the postprocessor to produce the demosaicked image pleasing for viewing. The interested reader can find the detailed description of the universal demosaicking framework and the two considered here solutions (Fig. 4) in [14]. 


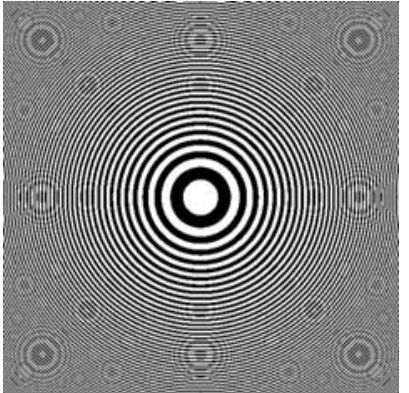

(a)

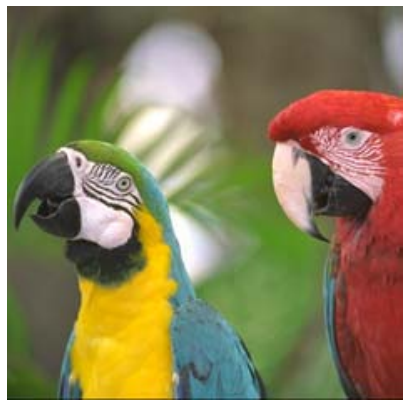

(c) (b)

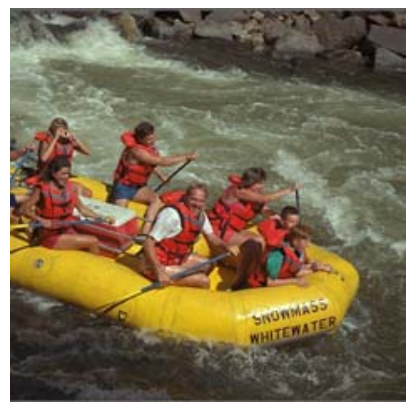

(d)

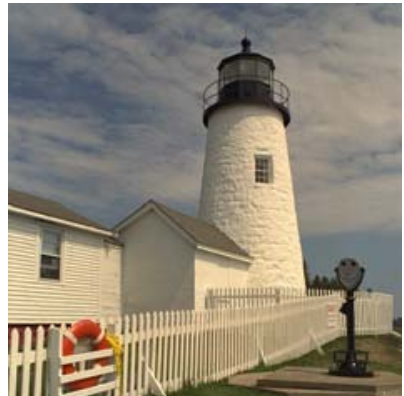

Fig. 5. Test images: (a) CZP, (b) Lighthouse, (c) Parrots, (d) Rafting.

\section{EXPERIMENTAL RESULTS}

To determine the performance of the CFAs listed in Fig. 2, a number of test images have been utilized. Examples such as the $512 \times 512$ artificial CZP image (Fig. 5a) and the $512 \times 512$ natural color images Lighthouse (Fig. 5b), Parrots (Fig. 5c) and Rafting (Fig. 5d) are used to emulate the application scenario. The test color images, which vary in both the complexity of the structural content (edges, fine details) and the color appearance, have been captured using three-sensor devices and normalized to 8-bit per channel RGB representation. Following the evaluation procedure depicted in Fig. 6, tests were performed by sampling the original images (Fig. 5) with each of the CFAs shown in Fig. 2 to obtain a CFA image $z$. Thus, the CFA image data $z_{(r, s)}$ is given by $z_{(r, s)}=o_{(r, s) 1}$ for $d_{(r, s) 1}=1, \quad z_{(r, s)}=o_{(r, s) 2}$ for $d_{(r, s) 2}=1$, and $z_{(r, s)}=o_{(r, s) 3}$ for $d_{(r, s) 3}=1$, where $d_{(r, s) k}$ denotes the location flags corresponding to the $k$-th color channel and $o_{(r, s) k}$ denotes the $\mathrm{R}(k=1), \mathrm{G}(k=2)$ and $\mathrm{B}(k=3)$ component of the original color pixel $\mathbf{o}_{(r, s)}=\left[o_{(r, s) 1}, o_{(r, s) 2}, o_{(r, s) 3}\right]$ occupying the location $(r, s)$, with $r=1,2, \ldots, K_{1}$ and $s=1,2, \ldots, K_{2}$. Using the two demosaicking solutions shown in Fig. 4, the demosaicked image $\mathbf{x}$ is generated by applying the universal demosaicking framework [14] onto the CFA image $z$. To evaluate the performance of the considered CFAs (Fig. 2), image quality was measured by comparing the original color image to the demosaicked image. To facilitate the objective comparisons, the RGB color space based mean absolute error (MAE) and mean square error (MSE) criteria, and the CIE-LUV color space based normalized color difference (NCD) criterion are used in this work. The interested reader can find the definitions of the above-listed criteria in [4].

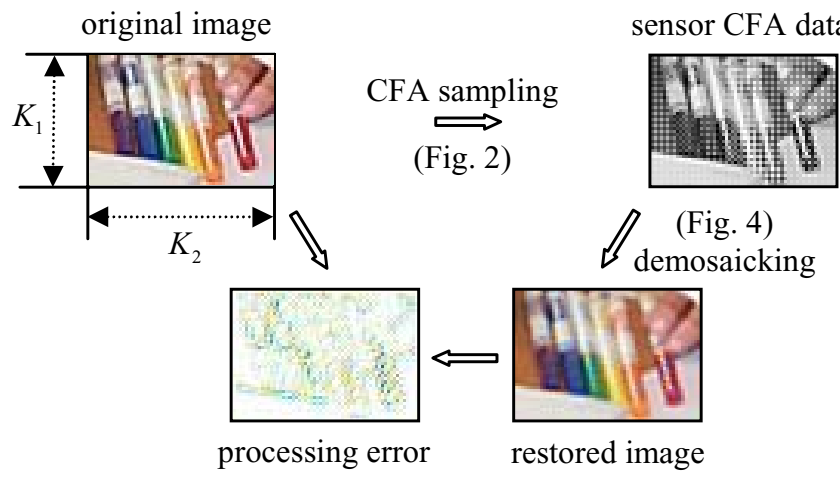

Fig. 6. Evaluation procedure.

Demosaicking results reported in Tables I and II show that the use of the diagonal stripe CFA (Fig. 2e) provides the best performance for the cost-effective demosaicking solution. Other CFAs suitable for this variant of the universal demosaicking framework are some of the periodic (Figs. 2a,c) and pseudo-random (Figs. 2g,i) CFAs. In the case of the sophisticated demosaicking solution, the Bayer (Fig. 2a), proposed here (Fig. 2c), diagonal stripe (Fig. 2e) and pseudorandom (Fig. 2g) CFA outperform other CFAs. Simple inspection of the results corresponding respectively to the costeffective (Table 1) and the sophisticated (Table 2) demosaicking solution shows an improvement obtained through the utilization of the edge-sensing mechanism, spectral model and the postprocessor during the processing.

Fig. 7 and Fig. 8 demonstrate the ability (or its lack) of both the CFA and the demosaicking solution to preserve various image frequencies. As shown in Fig. 7, each of the CFAs resulted in aliasing effects and color artifacts. For example, the images obtained using the CFAs shown in Figs. 2a,g,h,j suffer from aliasing in the horizontal, vertical, and diagonal direction. The use of the CFAs shown in Figs. 2b,d produced aliasing artifacts mainly in the horizontal direction, whereas CFAs in Figs. 2e,f,i and Fig. 2c resulted in aliasing mostly observed in the diagonal and vertical direction, respectively. By replacing the cost-effective demosaicked solution with its sophisticated variant, aliasing can be significantly reduced, if not removed altogether using some CFAs (Figs. 8a-c,i).

Fig. 9 and Fig. 10 depict enlarged parts of the natural color images cropped in areas with significant structural contents. Visual inspection of the demosaicked images reveals that the performance highly depends on the orientation and size of the edges and fine details in the area under consideration, and that the choice of the CFA plays an important role in obtaining the required visual quality. For example, although the costeffective demosaicking solution was used, the complex fence area in the image Lighthouse shown in Figs. 9c,e,f,i,j does not suffer so much from aliasing, which is even present in the images obtained using the sophisticated demosaicking solution combined with the well-known CFAs (Figs. 9a,b,d). In all cases shown in Figs. 9 and 10, images obtained using the sophisticated demosaicking solution show the enhanced color appearance and image sharpness. 
TABLE I

COMPARISON OF THE CFAS USING THE COST-EFFECTIVE DEMOSAICKING SOLUTION SHOWN IN FIG. 4A

\begin{tabular}{|c|c|c|c|c|c|c|c|c|c|c|c|c|}
\hline \multirow{2}{*}{$\frac{\text { Image }}{\text { CFA / Criterion }}$} & \multicolumn{3}{|c|}{ CZP (Fig. 5a) } & \multicolumn{3}{|c|}{ Lighthouse (Fig. 5b) } & \multicolumn{3}{|c|}{ Parrots (Fig. 5c) } & \multicolumn{3}{|c|}{ Rafting (Fig. 5d) } \\
\hline & MAE & MSE & NCD & MAE & MSE & NCD & MAE & MSE & $\mathrm{NCD}$ & MAE & MSE & NCD \\
\hline Fig. 2a & 39.49 & 3457.7 & 0.8977 & 4.92 & 137.9 & 0.0580 & 2.21 & 32.8 & 0.0269 & 4.93 & 94.0 & 0.0750 \\
\hline Fig. $2 b$ & 42.76 & 4005.7 & 0.9461 & 5.57 & 172.1 & 0.0655 & 2.38 & 37.7 & 0.0293 & 5.24 & 105.8 & 0.0801 \\
\hline Fig. $2 \mathrm{c}$ & 41.50 & 3815.5 & 0.9496 & 5.23 & 144.9 & 0.0615 & 2.32 & 37.7 & 0.0292 & 5.35 & 107.7 & 0.0840 \\
\hline Fig. $2 \mathrm{~d}$ & 45.94 & 6430.3 & 1.2728 & 6.96 & 307.0 & 0.0983 & 3.14 & 68.0 & 0.0475 & 6.00 & 153.3 & 0.1109 \\
\hline Fig. $2 \mathrm{e}$ & 36.37 & 3314.7 & 0.8297 & 4.81 & 117.8 & 0.0545 & 2.14 & 30.2 & 0.0268 & 4.91 & 92.9 & 0.0772 \\
\hline Fig. $2 \mathrm{f}$ & 37.31 & 4330.0 & 0.9784 & 5.16 & 146.2 & 0.0653 & 2.42 & 43.9 & 0.0325 & 5.39 & 116.9 & 0.0906 \\
\hline Fig. $2 g$ & 39.92 & 3671.8 & 0.9289 & 5.06 & 144.0 & 0.0606 & 2.22 & 34.2 & 0.0276 & 5.06 & 99.5 & 0.0788 \\
\hline Fig. $2 \mathrm{~h}$ & 42.85 & 4524.8 & 1.0322 & 5.60 & 176.7 & 0.0698 & 2.50 & 45.5 & 0.0334 & 5.53 & 120.2 & 0.0913 \\
\hline Fig. $2 \mathrm{i}$ & 36.79 & 3670.1 & 0.9308 & 4.91 & 131.2 & 0.0642 & 2.27 & 35.6 & 0.0311 & 5.05 & 99.0 & 0.0869 \\
\hline Fig. $2 \mathrm{j}$ & 42.96 & 4545.2 & 1.0437 & 5.44 & 164.5 & 0.0679 & 2.54 & 47.6 & 0.0335 & 5.63 & 125.1 & 0.0923 \\
\hline
\end{tabular}

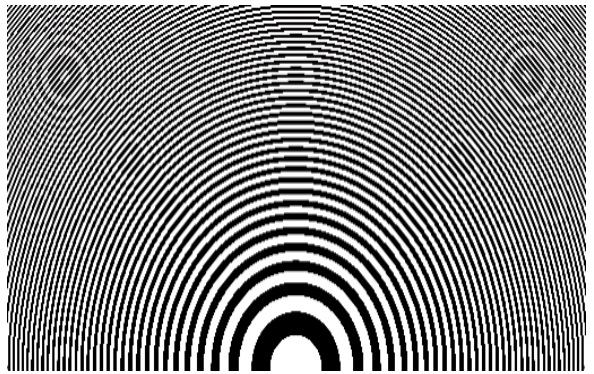

(o)

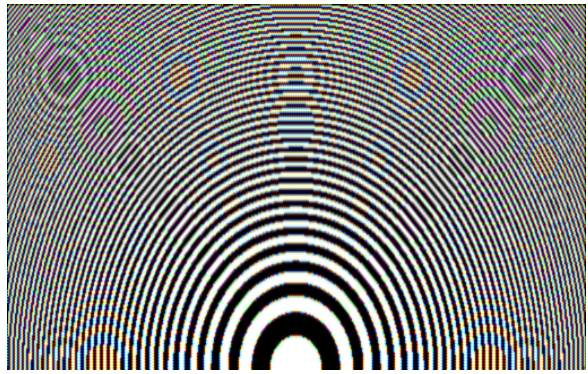

(a)

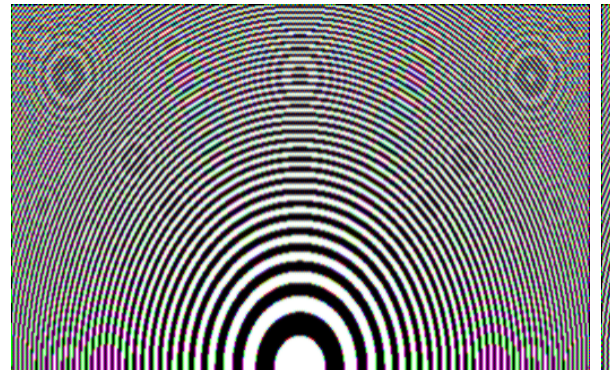

(b)

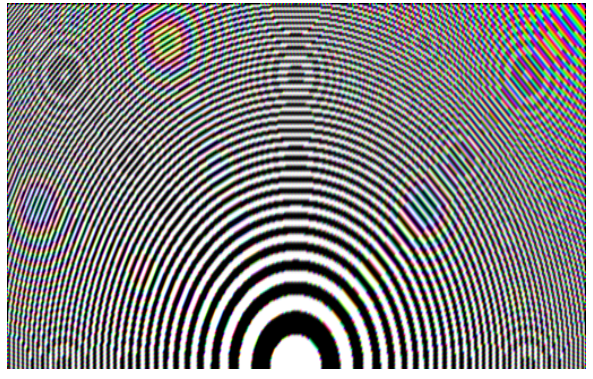

(e)

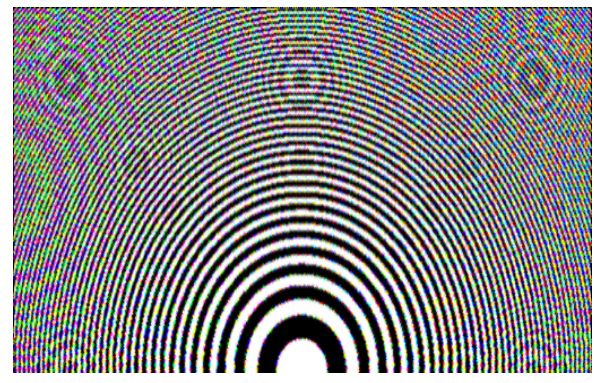

(h)

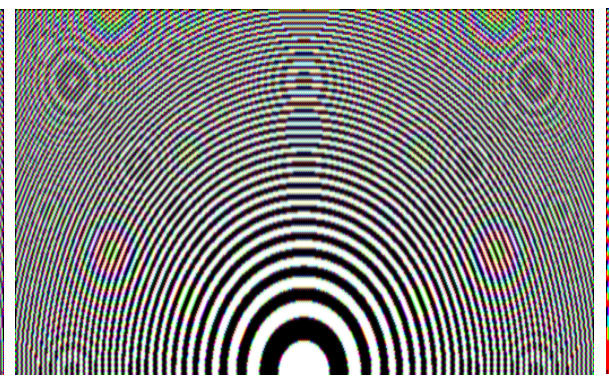

(c)

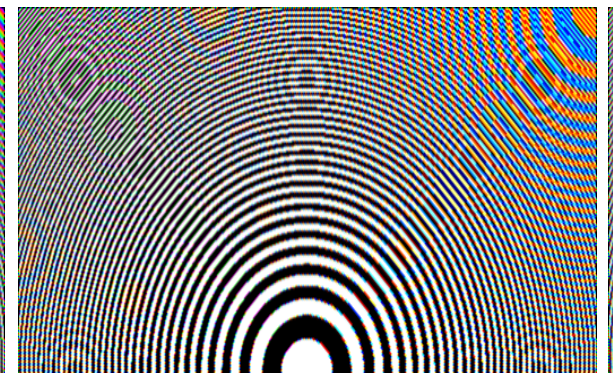

(f)

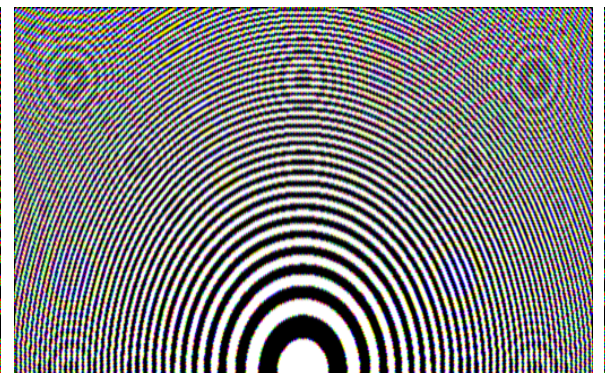

(i)

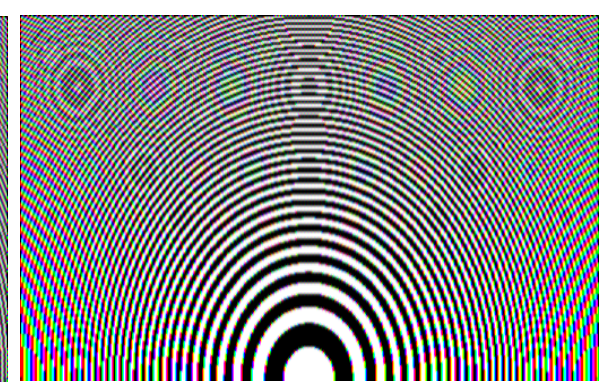

(d)

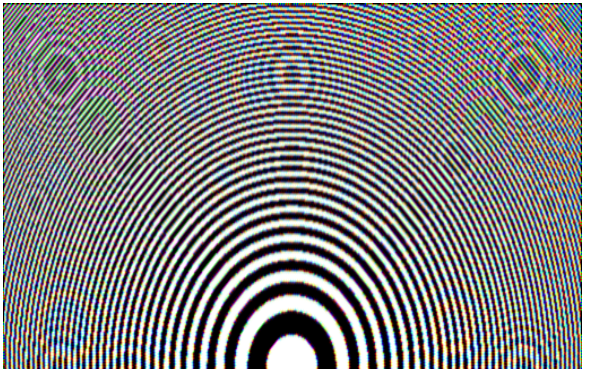

(g)

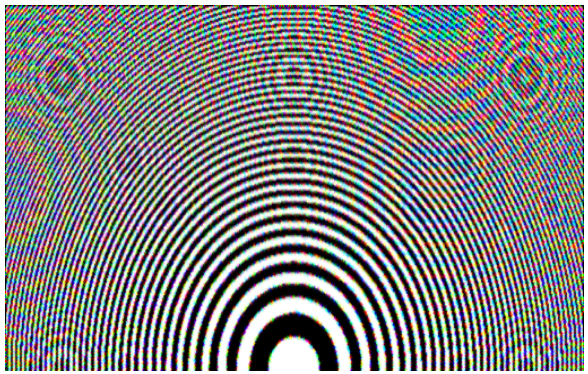

(j)

Fig. 7. CZP image demosaicking using the cost-effective solution in Fig. 4a: (o) original image, (a-j) demosaicked images corresponding to the CFAs shown in Figs. 2a-j, respectively. 
TABLE II

COMPARISON OF THE CFAS USING THE SOPHISTICATED DEMOSAICKING SOLUTION SHOWN IN FIG. 4B

\begin{tabular}{|c|c|c|c|c|c|c|c|c|c|c|c|c|}
\hline Image & \multicolumn{3}{|c|}{ CZP (Fig. 5a) } & \multicolumn{3}{|c|}{ Lighthouse (Fig. 5b) } & \multicolumn{3}{|c|}{ Parrots (Fig. 5c) } & \multicolumn{3}{|c|}{ Rafting (Fig. 5d) } \\
\hline CFA / Criterion & MAE & MSE & $\mathrm{NCD}$ & MAE & MSE & $\mathrm{NCD}$ & MAE & MSE & $\mathrm{NCD}$ & MAE & MSE & $\mathrm{NCD}$ \\
\hline Fig. 2a & 2.37 & 33.9 & 0.1439 & 1.70 & 13.7 & 0.0230 & 1.17 & 5.5 & 0.0169 & 1.95 & 18.2 & 0.0350 \\
\hline Fig. 2b & 3.98 & 78.9 & 0.2252 & 2.16 & 24.4 & 0.0306 & 1.29 & 7.1 & 0.0182 & 2.20 & 23.0 & 0.0394 \\
\hline Fig. 2c & 3.32 & 69.1 & 0.1893 & 1.75 & 12.7 & 0.0247 & 1.24 & 6.5 & 0.0178 & 2.28 & 24.1 & 0.0412 \\
\hline Fig. 2d & 19.86 & 2375.3 & 0.5889 & 5.69 & 251.7 & 0.0773 & 2.81 & 48.2 & 0.0389 & 4.43 & 94.5 & 0.0782 \\
\hline Fig. 2e & 6.30 & 279.2 & 0.2368 & 1.76 & 12.3 & 0.0248 & 1.31 & 7.7 & 0.0182 & 2.43 & 25.7 & 0.0432 \\
\hline Fig. $2 f$ & 5.26 & 180.9 & 0.2351 & 2.08 & 18.9 & 0.0282 & 1.43 & 10.7 & 0.0200 & 2.86 & 34.3 & 0.0494 \\
\hline Fig. $2 g$ & 2.75 & 48.2 & 0.1705 & 1.87 & 15.3 & 0.0252 & 1.16 & 6.0 & 0.0167 & 2.15 & 21.2 & 0.0382 \\
\hline Fig. $2 \mathrm{~h}$ & 8.24 & 372.4 & 0.3406 & 2.89 & 46.4 & 0.0392 & 1.57 & 13.0 & 0.0225 & 2.95 & 37.0 & 0.0527 \\
\hline Fig. $2 \mathrm{i}$ & 5.88 & 185.6 & 0.2658 & 2.13 & 19.7 & 0.0308 & 1.35 & 8.9 & 0.0198 & 2.60 & 27.6 & 0.0481 \\
\hline Fig. $2 \mathrm{j}$ & 6.24 & 220.6 & 0.2851 & 2.36 & 27.1 & 0.0322 & 1.58 & 13.0 & 0.0222 & 2.90 & 37.0 & 0.0507 \\
\hline
\end{tabular}

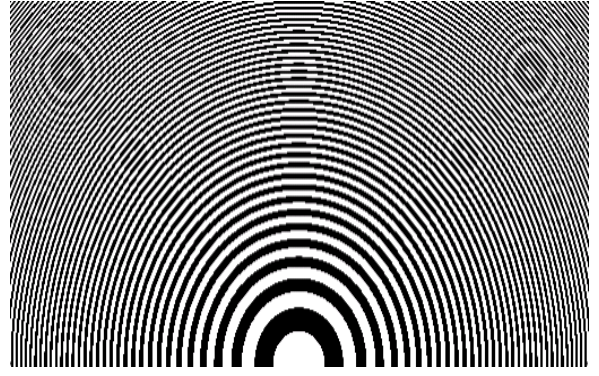

(o)

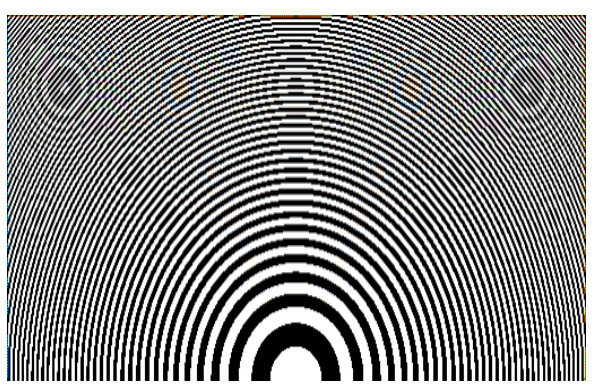

(a)

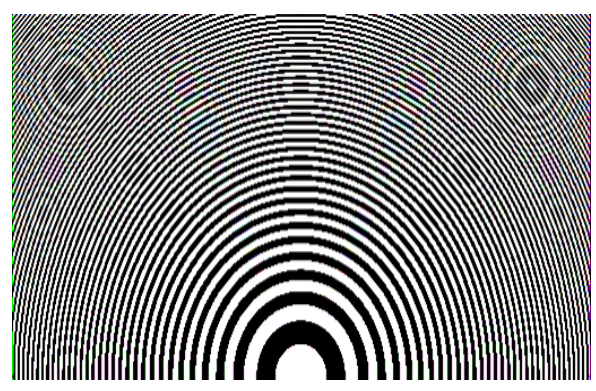

(b)

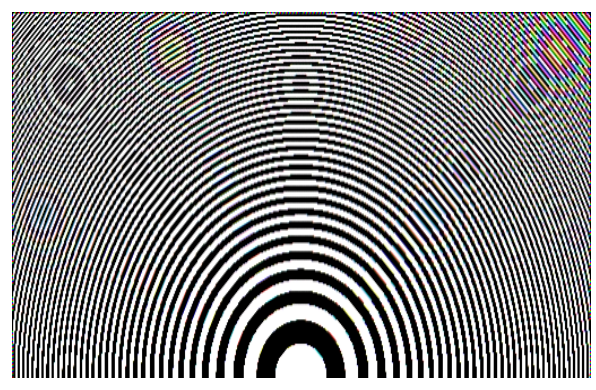

(e)

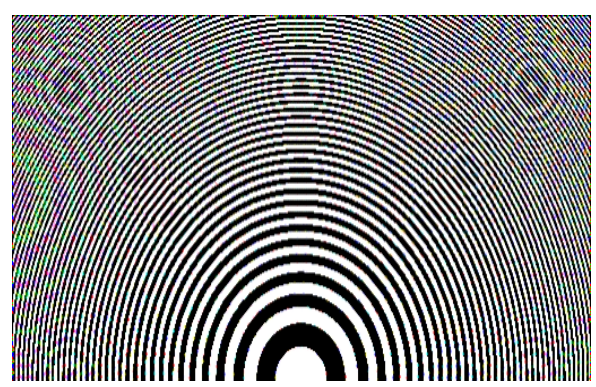

(h)

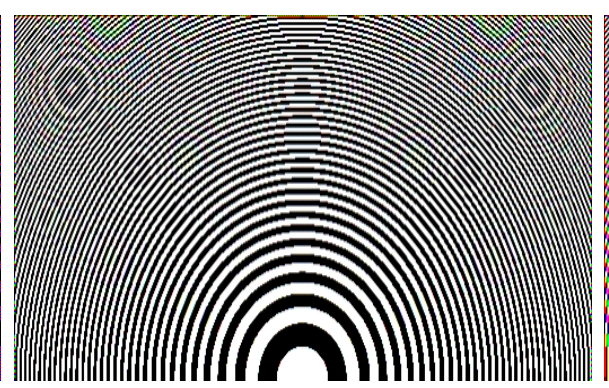

(c)

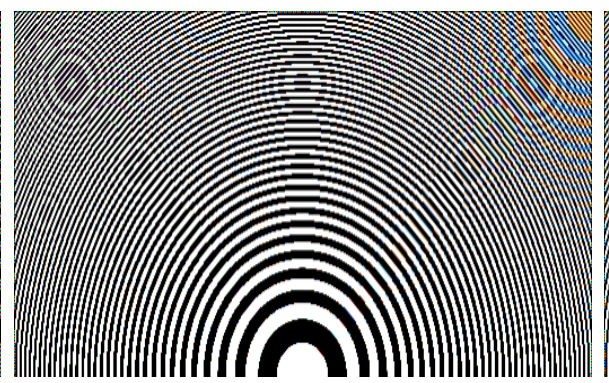

(f)

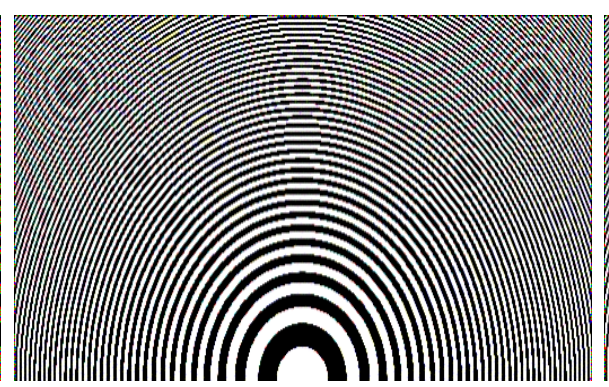

(i)

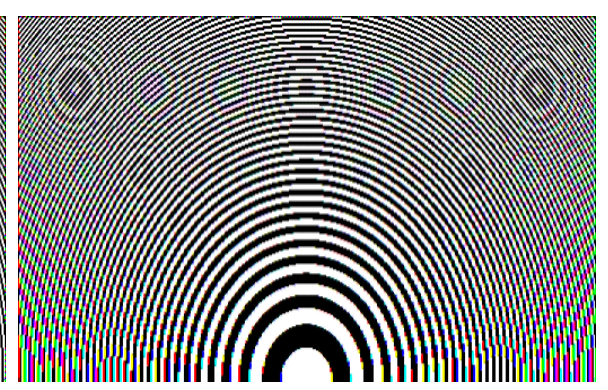

(d)

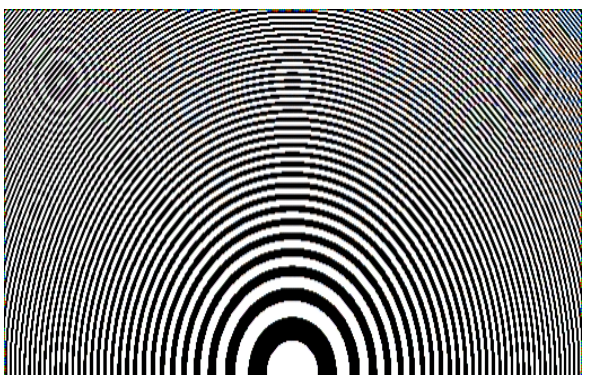

(g)

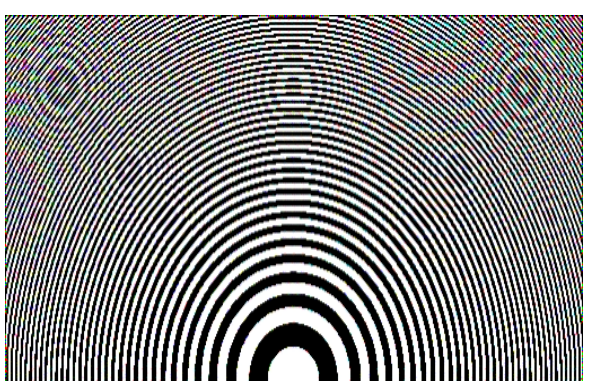

(j)

Fig. 8. CZP image demosaicking using the sophisticated solution in Fig. 4b: (o) original image, (a-j) demosaicked images corresponding to the CFAs shown in Figs. 2a-j, respectively. 
(a)

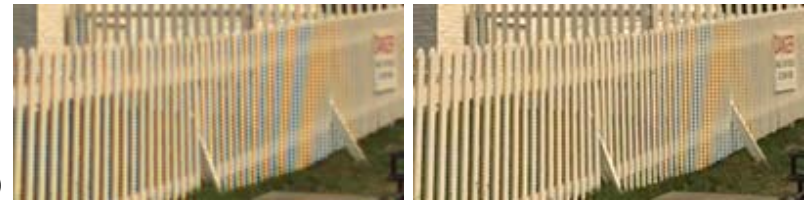

(b)

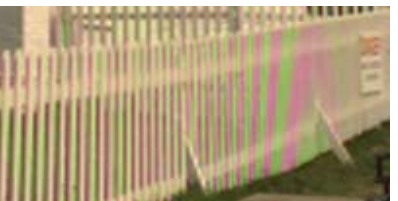

(c)

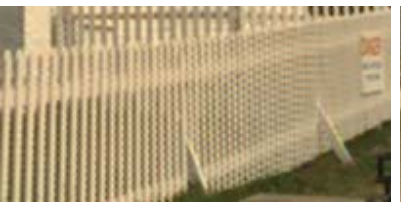

(d)

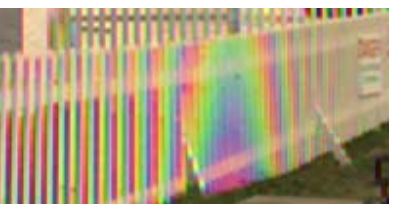

(e)

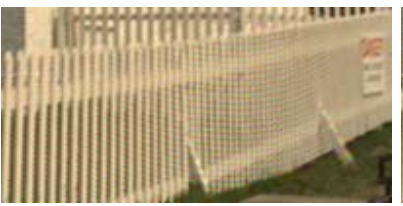

(f)

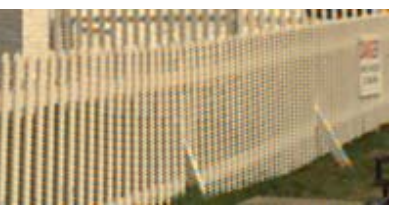

(g)

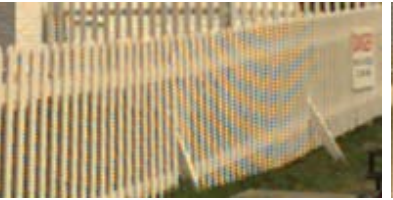

$$
\text { Iowithms }
$$

Antin:

(h)
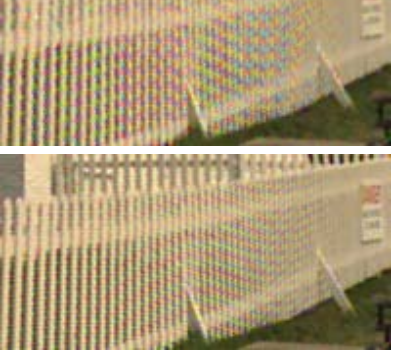

(j)

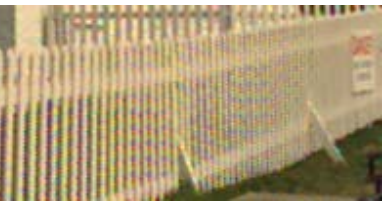

Fig. 9. Lighthouse image demosaicking: (o) original image, (a-j) demosaicked images corresponding to the CFAs shown in Figs. 2a-j, respectively. Demosaicking was performed using: (left column) costeffective solution, (right column) sophisticated solution.
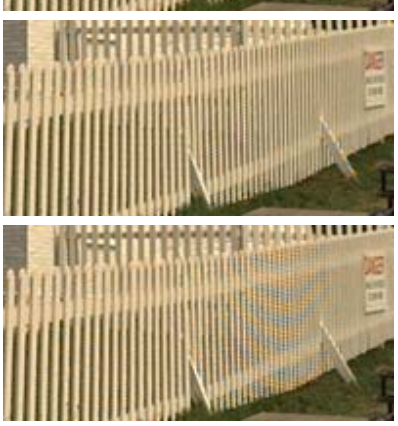

(o)

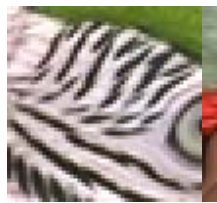

(a)

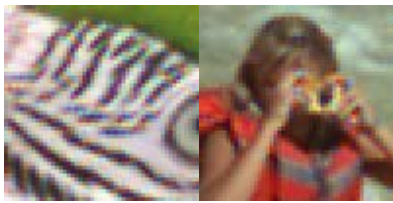

(b)

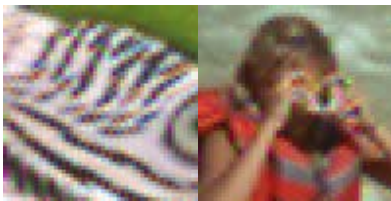

(c)

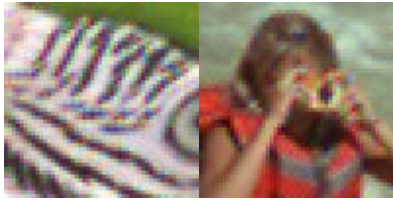

(d)

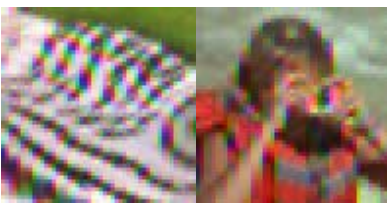

(e)

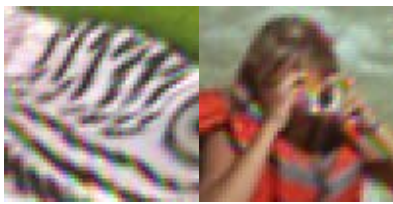

(f)

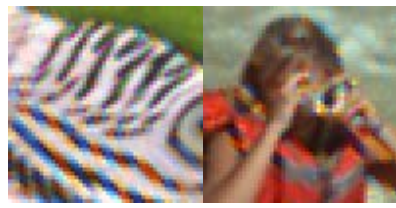

(g)

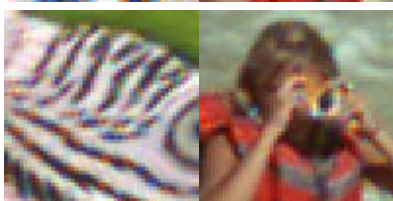

(h)
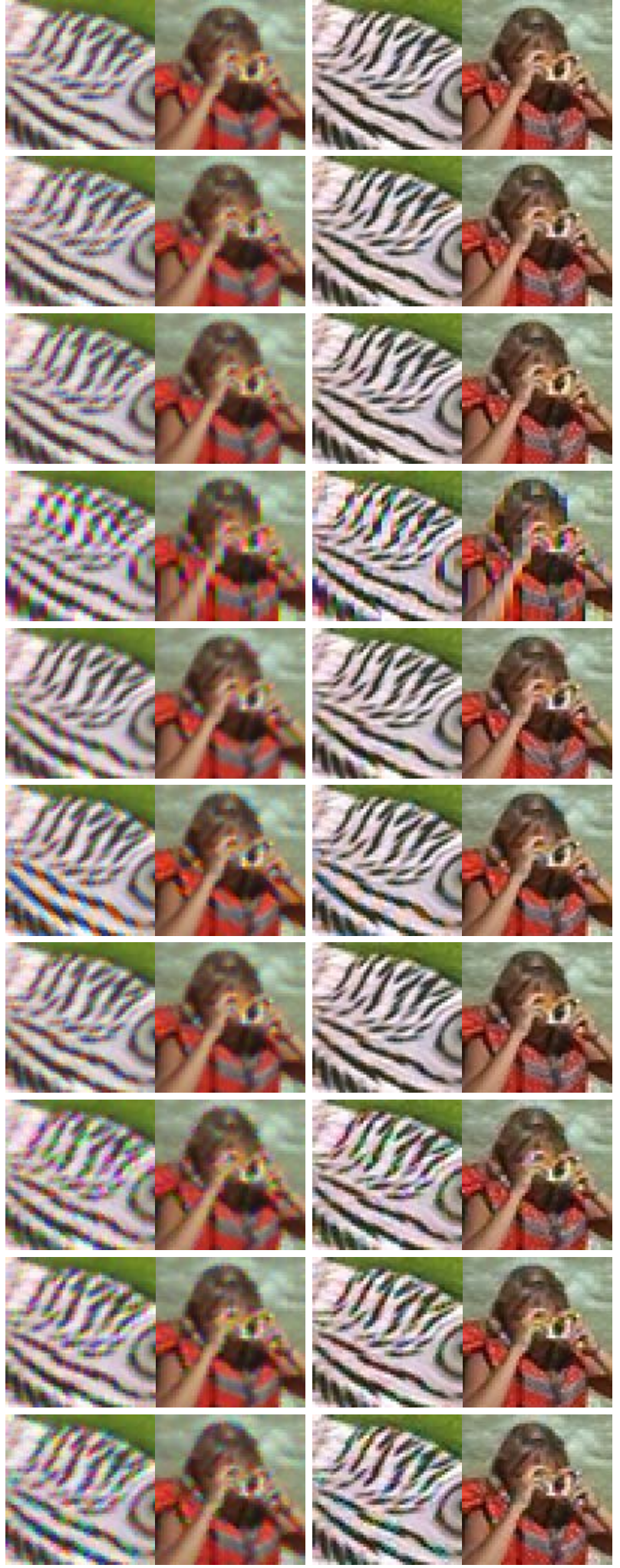

Fig. 10. Parrots and Rafting image demosaicking: (o) original image, (a-j) demosaicked images corresponding to the CFAs shown in Figs. 2a-j, respectively. Demosaicking was performed using: (left column) costeffective solution, (right column) sophisticated solution. 
In the summary, the following conclusions can be drawn: i) the perfect CFA does not exist, ii) the choice of the CFA greatly influences the performance of the single-sensor imaging pipeline, and iii) visual impairments can be reduced by changing the CFA and/or utilizing the sophisticated demosaicking solution with the included postprocessor.

\section{Conclusion}

This paper presented the design, analysis and performance evaluation of the color filter array (CFA) which is the most crucial element in the single-sensor consumer electronic device used to capture the visual scene. Of particular interest were RGB CFAs due to the relative simplicity of the demosaicking process and the natural connection to the commonly used RGB format for displaying and storage of the captured images. The universal demosaicking framework was used to guide the performance evaluation of the ten RGB CFAs separately employed in the single-sensor imaging pipeline. Experimentation performed here suggests that the choice of the CFA critically affects the amount of various visual impairments, such as color shifts, artifacts, and aliasing effects, present in the demosaicked image.

\section{REFERENCES}

[1] K. Parulski and K. E. Spaulding, "Color image processing for digital cameras," in Digital Color Imaging Handbook, (eds.) G. Sharma, CRC Press, Boca Raton, FL., pp. 728-757, 2002.

[2] P. L. P. Dillon, D. M. Lewis, and F. G. Kaspar, "Color imaging system using a single CCD area array," IEEE Journal of Solid-State Circuits, vol. 13, no. 1, pp. 28-33, February 1978.

[3] T. Lule, S. Benthien, H. Keller, et al., "Sensitivity of CMOS based imagers and scaling perspectives," IEEE Transactions on Electron Devices, vol. 47, no. 11, pp. 2110-2122, November 2000.

[4] R. Lukac and K. N. Plataniotis, "Data-adaptive filters for demosaicking: A framework," IEEE Transactions on Consumer Electronics, vol. 51, no. 2, pp.560-570, May 2005.

[5] Technical Committee ISO/TC 42, Photography, "Electronic still picture imaging - Removable memory, Part 2: Image data format - TIFF/EP," ISO 12234-2, January 2001.

[6] R. Lukac and K. N. Plataniotis, " Fast video demosaicking solution for mobile phone imaging applications," IEEE Transactions on Consumer Electronics, vol. 51, no. 2, pp. 675-681, May 2005.

[7] $\mathrm{X} . \mathrm{Wu}$ and N. Zhang, "Primary-consistant soft-decision color demosaicking for digital cameras," IEEE Transactions on Image Processing, vol. 13, no. 9, pp. 1263-1274, September 2004.

[8] B. K. Gunturk, J. Glotzbach, Y. Altunbasak, R. W. Schaffer, and R. M. Murserau, "Demosaicking: color filter array interpolation," IEEE Signal Processing Magazine, vol. 22, no. 1, pp. 44-54, January 2005.

[9] R. Lukac, B. Smolka, K. Martin, K. N. Plataniotis, and A. N. Venetsanopulos, "Vector filtering for color imaging," IEEE Signal Processing Magazine; vol. 22, no. 1, pp. 74-86, January 2005.

[10] B. E. Bayer, "Color imaging array," U.S. Patent 3971 065, July 1976.

[11] S. Yamanaka, "Solid state camera," U.S. Patent 4054 906, Nov. 1977.

[12] FillFactory, "Technology image sensor: the color filter array," Available Online: http://www.fillfactory.com/htm/technology/htm/rgbfaq.htm.

[13] M. Parmar and S.J. Reeves, "A perceptually based design methodology for color filter arrays," in Proc. IEEE Int. Con. Acoustics, Speech, and Signal Processing (ICASSP'04), vol. 3, pp. 473-476, May 2004.

[14] R. Lukac and K. N. Plataniotis, " Universal demosaicking for imaging pipelines with an RGB color filter array," Pattern Recognition, vol. 38, no. 11, pp. 2208-2212, November 2005.

[15] R. Lukac and K. N. Plataniotis, "Normalized color-ratio modeling for CFA interpolation," IEEE Transactions on Consumer Electronics, vol. 50, no. 2, pp. 737-745, May 2004.
[16] J. Adams, K. Parulski, and K. Spaulding, "Color processing in digital cameras," IEEE Micro, vol. 18, no. 6, pp. 20-30, Nov./Dec. 1998.

[17] R. Lukac, K. Martin, and K. N. Plataniotis, "Demosaicked image postprocessing using local color ratios," IEEE Transactions on Circuit and Systems for Video Technology, vol. 14, no. 6, pp. 914-920, June 2004.

[18] S.C. Pei and I.K. Tam, "Effective color interpolation in CCD color filter arrays using signal correlation," IEEE Trans. Circuits and Systems for Video Technology, vol. 13, no. 6, pp. 503-513, June 2003.

[19] W. Lu and Y. P. Tang, "Color filter array demosaicking: new method and performance measures," IEEE Transactions on Image Processing, vol. 12, no. 10, pp. 1194-1210, October 2003.

[20] R. Lukac, K. N. Plataniotis, D. Hatzinakos, and M. Aleksic, "A novel cost effective demosaicing approach," IEEE Transactions on Consumer Electronics, vol. 50, no. 1, pp. 256-261, February 2004.

[21] D. Alleyson, S. Susstrunk and J. Herault, "Linear demosaicing inspired by the human visual system," IEEE Transactions on Image Processing, vol. 14, no. 4, pp. 439-449, April 2005

[22] N. Kehtarnavaz, H.J Oh, and Y. Yoo, "Color filter array interpolation using color correlations and directional derivatives," Journal of Electronic Imaging, vol. 12, no. 4, pp. 621-632, October 2003.

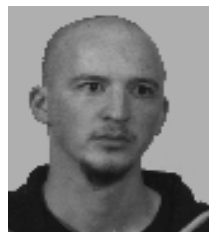

Rastislav Lukac received the M.S. (Ing.) and Ph.D. degrees in Telecommunications from the Technical University of Kosice, Slovak Republic in 1998 and 2001, respectively. From February 2001 to August 2002 he was an Assistant Professor at the Department of Electronics and Multimedia Communications at the Technical University of Kosice. Since August 2002 he is a Researcher in Slovak Image Processing Center in Dobsina, Slovak Republic. From January 2003 to March 2003 he was a Postdoctoral Fellow at the Artificial Intelligence \& Information Analysis Lab at the Aristotle University of Thessaloniki, Greece. Since May 2003 he has been a Post-doctoral Fellow with the Edward S. Rogers Sr. Department of Electrical and Computer Engineering at the University of Toronto in Toronto, Canada. His research interests include digital camera image processing, microarray image processing, multimedia security, and nonlinear filtering and analysis techniques for color image \& video processing.

Dr. Lukac is a Member of the IEEE Circuits and Systems, IEEE Consumer Electronics, and IEEE Signal Processing Societies. He serves as a Technical Reviewer for various scientific journals and he participates as a Member of numerous International Conference Committees. In 2003 he was awarded the NATO/NSERC Science Award.

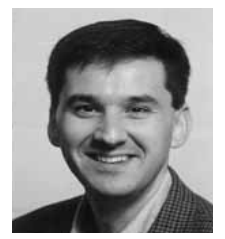

Konstantinos N. Plataniotis received the B. Engineering degree in Computer Engineering from the Department of Computer Engineering and Informatics, University of Patras, Patras, Greece in 1988 and the M.S and $\mathrm{Ph} . \mathrm{D}$ degrees in Electrical Engineering from the Florida Institute of Technology (Florida Tech), Melbourne, Florida in 1992 and 1994 respectively. From August 1997 to June 1999 he was an Assistant Professor with the School of Computer Science at Ryerson University. He is currently an Associate Professor at the Edward S. Rogers Sr. Department of Electrical \& Computer Engineering where he researches and teaches image processing, adaptive systems, and multimedia signal processing. He co-authored, with A.N. Venetsanopoulos, a book on "Color Image Processing \& Applications", Springer Verlag, May 2000, he is a contributor to seven books, and he has published more than 300 papers in refereed journals and conference proceedings in the areas of multimedia signal processing, image processing, adaptive systems, communications systems and stochastic estimation.

Dr. Plataniotis is a Senior Member of IEEE, an Associate Editor for the IEEE Transactions on Neural Networks, a past member of the IEEE Technical Committee on Neural Networks for Signal Processing. He was the Technical Co-Chair of the Canadian Conference on Electrical and Computer Engineering (CCECE) 2001, and CCECE 2004. He is the Vice-chair of the 2006 IEEE International Conference in Multimedia and Expo (ICME 2006), the Technical Program Co-chair for 2006 IEEE Intelligent Transportation Systems Conference (ITSC 2006), and the Image Processing Area Editor for the IEEE Signal Processing Society e-letter. 